\title{
Function-Space Assignment for Educational Facilities
}

\author{
Ren-Jye Dzeng, Chong-Wey Lin, and Fan-Yi Hsiao
}

\begin{abstract}
Function-space assignment, which allocates a function for each space in a facility, is one of the most important factors in determining the usability performance of a facility. An educational facility is often used through activity participation of different groups such as faculty or construction management graduate students. Members of a group participate in the same activities but with different behaviors (e.g., attendance rate, arrival time). This research uses two modules to optimize the function space assignment. The first module uses simulation technique to randomly generate activity data to accommodate the uncertain nature of activities. The second module uses the fmGA to optimize the function-space assignment. This paper also presents a real case study to demonstrate the use of the proposed model and compares its assignments with those generated by a renovation architect. The result shows a $14.80 \%$ higher objective value than the architect's version.
\end{abstract}

Index Terms-Function-space assignment, fast messy genetic algorithms, activity simulation.

\section{INTRODUCTION}

A building design with a good level of service relies on the full understanding of occupants' needs and activities, and being able to create a design that accommodates their needs. Assuming the occupants' needs and activities are known, in most cases the only way the designers can verify their design is to let the design checked by the client representative or other design experts. Because no appropriate tool is available, currently the verification or assessment of the design during the design phase is still difficult.

Buildings play a role of accommodating occupants' organizations and equipment, and enable their activities. The relation between the occupants and the building is mediated through the functional spaces where the activities take place [1]. Moreover, the level of service of indoor space in a building depends on whether the design of functional spaces assignments, corridors and stairs are satisfied the activities needs of each occupant. For example, in an educational building, there are several types of occupants (e.g. undergraduates, graduate students and professors), and they move around the different functional spaces (e.g. classroom, library, laboratory, meeting room and administration office), corridors and stairs in the building based on the activities which they have been formally assigned (e.g. teaching,

Manuscript received October 9, 2012; revised January 2, 2013. This work was partially supported by the National Science Council grants (NSC 99-2221-E-009-133-MY3). Their support is greatly appreciated.

R. J. Dzeng and F. Y. Hsiao are with the Department of Civil Engineering, National Chiao-Tung University, 1001, University Road, Hsin-Chu, Taiwan (e-mails:rjdzeng@mail.nctu.edu.tw, minlove.xiao@gmail.com).

C. W. Lin is with the Department of Commutation and Technology, National Chiao-Tung University, 1001, University Road, Hsin-Chu, Taiwan (e-mail: cwlin@mail.nctu.edu.tw). meetings, and office work) or in which they are personally interested (e.g. personal research, community activity).

This research uses two modules to optimize the function space assignment. The first module uses simulation technique to randomly generate activity data to accommodate the uncertain nature of activities. The second module uses the fmGA to optimize the function-space assignment. This paper also presents a real case study to demonstrate the use of the proposed model and compares its assignments with those generated by a renovation architect.

\section{Problem Solving Techniques for Facility Layout}

In architecture, optimization techniques have been used primarily for solving problems of facility layout, structural design, and building performance [2]. Facility layout optimization is concerned with finding feasible topology and dimensions of interrelated objects that meet all design requirements and maximize design preferences [3]. Previous research has developed several formulations for optimizing facility layout problems. For discrete formulations, the quadratic assignment problems (QAP) [4] is the most commonly encountered in the literature.

For the QAP, several types of problem-solving approaches have been proposed, such as exact, heuristics and meta-heuristics approaches. As the QAP is one of the most difficult problems in the NP-hard class. Exact approaches are generally unable to solve problems of size larger than $n=15$ [5]. Since exact solutions require large expenditures of time and money, it may not be worthwhile to search the optimum solutions except for the rare circumstances [6]. For this reason, several heuristics and meta-heuristics approaches have been developed to search sub-optimal solutions within a reasonable time limit.

The simple genetic algorithm (sGA) is one of the meta-heuristics approaches. First developed by Holland in 1975 [7], sGA was an efficient and popular algorithm. Goldberg et al. [8] subsequently developed the messy genetic algorithm (mGA) in 1989 in order to improve sGA weaknesses. Several experiments have proven the mGA much better at solving permutation problems than sGA. In 1993, Goldberg et al. [9] developed the fast messy genetic algorithm (fmGA) to reduce the high memory consumption of operation processes. Over the years, mGAs and fmGA have been used successfully in water distribution system design, ready mixed concrete trucks dispatching, design of fuzzy control systems, solution of clustering problems, and learning classifier systems. For its advantage, we used fmGA to search the optimal solution for this research.

\section{RESEARCH PROBLEM}

Consider a scenario of designing a new educational facility 
or remodeling an existing one whose spaces, functions (e.g., large classroom, small classroom, administration office, meeting room), and activities (e.g., attending "Construction Management" class, reading newspaper in the department library) are known.

Each space at each time slot can only be assigned with one activity. Given a predetermined layout of the spaces, the objective is to assign functions to the spaces so that the maximum service level can be achieved. Although activities are assumed to be known, some of their attributes may be of uncertain nature. For example, a student is known to attend the "Construction Management" class, but the actual arrival time may be probabilistic. The actual frequency of going to the department library may be also probabilistic.

Given the spaces and functions data, as shown in Fig. 1, this research uses two modules to optimize the function space assignment. The first module uses simulation technique to randomly generate activity data to accommodate the uncertain nature of activities. The second module uses a genetic algorithm to optimize the function-space assignment.

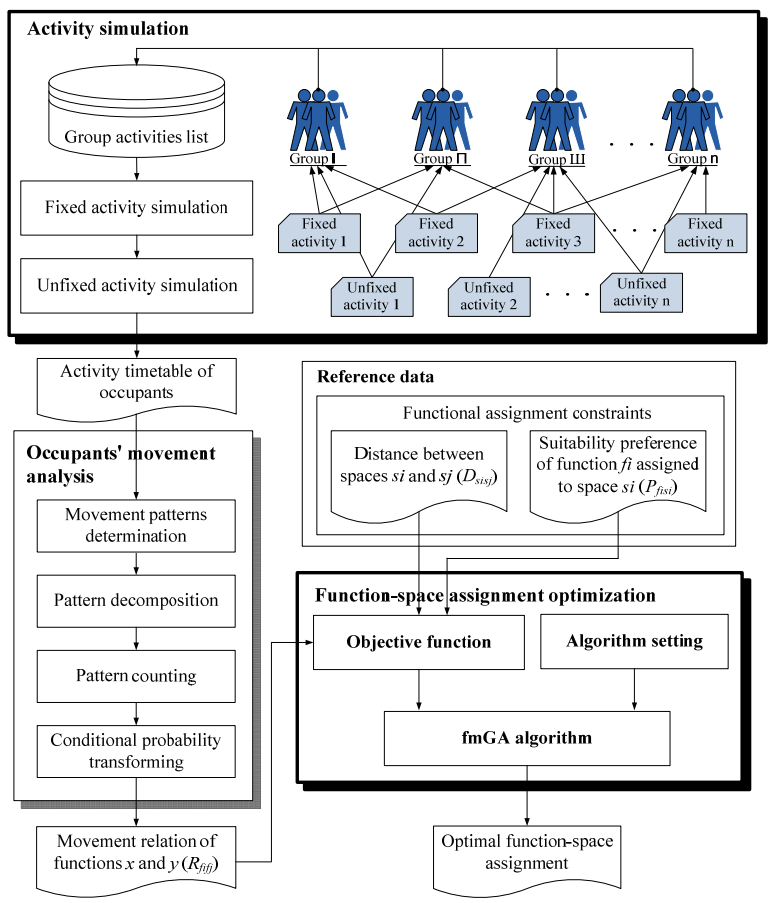

Fig. 1. The proposed model.

\section{The Proposed ModeL}

\section{A. Modeling of Group and Activities}

Occupants in an educational facility may share similarity in the activities they participate in. For example, the graduate students of construction management Master's program can be defined as a group. Members of a group have the same set of activities they may participate in and their activity participating behaviors are similar. Table I lists the attributes of a group. The name and ID describe the group. The number-of-members determine the number of occupants generated during the simulation. The activity-participating-list is a list of what activity and how the group member participates in. Each list item is an activity with a list of behavior attributes.

Doherty [10] stated that occupants tend to schedule their activities in a priority base, rather than time sequential way, that is, the high priority activities was top scheduled, the low priority activities was scheduled when having available time. Based on the argument, this research defines two types of activities, i.e., the fixed and unfixed activities. The fixed activity is an activity whose schedule is predetermined and fixed weekly. Examples are attending a requisite or elective class, a lab or a seminar. The unfixed activities include flexible activity and ad-hoc activity. Meeting with an advisor is a flexible activity because appointments need to be made but the meeting times may be different each week. The ad-hoc activity is an activity that an occupant carries out on his/her own accord without particular planning. Examples of ad-hoc activities are reading newspaper in the department library, or getting mail at the department office.

\begin{tabular}{ll}
\hline \hline group-name & Junior students \\
\hline group-ID & $\mathrm{G} 001$ \\
\hline number-of-members & 95 \\
\hline activity-participating-list & [(Act ID, behavior attributes)+] \\
\hline \hline
\end{tabular}

Table $\Pi$ lists the attributes of an activity. The schedule-specs attribute specifies the occurring day, time, and duration of the activity. For a fixed activity, the schedule-specs value is deterministic and can be a list of specification. For an unfixed activity, the schedule-specs value is probabilistic and the specification is composed of a list of perspective weekdays, possible start time range, possible duration range, and possible occurring frequency range.

Table $\amalg$ lists the behavior attributes, which describe how a group member participates in an activity. The attendance-rate is an average percentage of attendance of an occupant pertaining to an activity. The activity-arrival-trend and the activity-leaving-trend attributes are used to describe probabilistically the actual arrival and leaving times pertaining to an activity during the simulation.

TABLE П: ACTIVITY ATTRIBUTES

\begin{tabular}{|c|c|c|c|c|}
\hline \multirow{2}{*}{ Activity Attributes } & \multicolumn{2}{|c|}{ Fixed Activity } & \multicolumn{2}{|l|}{ Unfixed Activity } \\
\hline & Data type & Property & Data type & Property \\
\hline schedule-specs & [weekday, time, duration] + & \multirow{3}{*}{ Deterministic } & [(weekday-list), (time-range), (duration-range), (frequency-range)] & \multirow[t]{2}{*}{ Probabilistic } \\
\hline location & space-ID & & [space-ID- list] & \\
\hline repetition- period & [start date, end date] & & [start date, end date] & Deterministic \\
\hline
\end{tabular}


TABLE Ш: BEHAVIOR ATTRIBUTES

\begin{tabular}{ll}
\hline \multicolumn{1}{c}{ Behavior Attributes } & \multicolumn{1}{c}{ Data type } \\
\hline attendance-rate & rate $\%$ \\
\hline activity-arrival- trend & probability distribution \\
\hline activity-leaving -trend & probability distribution \\
\hline preferred-shorter-free-time & $\mathrm{Y} / \mathrm{N}$ \\
\hline \hline
\end{tabular}

Table IV shows examples of fixed activity and unfixed activity. For example, the members of a group may participate in a fixed activity "Attending Construction Management class" starting at 10:00AM Tuesday, 10:00AM Thursday, and 11:00AM Thursday, respectively. Each class lasts for 50 minutes at space F001 once a week for the period from Sep. $18^{\text {th }}, 2012$ to Jan. $24^{\text {th }}$, 2013. The average attendance rate of the group for this activity is 0.95 . The arrival time of attending the Tuesday class and the first Thursday class for the group is a normal distribution with mean equal to 5 minutes $(-10 \% \times 50=5)$ ahead of class start time, and standard deviation of 2.5 minutes $(-5 \% \times 50=2.5)$. The arrival time of attending the second Thursday class is a normal distribution with mean equal to 2.5 minutes $(-5 \% \times 50$ $=2.5)$ ahead of class start time, and standard deviation of 1 minutes $(-2 \% \times 50=1)$. The specification shows that students are more punctual for the second class in consecutive classes.

TABLE IV: EXAMPLES OF FIXED ACTIVITY AND UNFIXED ACTIVITY

\begin{tabular}{|c|c|c|c|c|c|}
\hline & & \multicolumn{2}{|r|}{ Fixed Activity } & \multicolumn{2}{|c|}{ Unfixed Activity } \\
\hline \multirow{2}{*}{\multicolumn{2}{|c|}{ Activity Example }} & ID & Name & ID & Name \\
\hline & & $\begin{array}{c}\text { F00 } \\
1\end{array}$ & $\begin{array}{l}\text { Attending Construction } \\
\text { Management class }\end{array}$ & U001 & $\begin{array}{l}\text { Reading } \\
\text { in the library }\end{array}$ \\
\hline \multirow[t]{3}{*}{ Activity Attributes } & schedule-specs & \multicolumn{2}{|c|}{$\begin{array}{l}\text { [THU, 10:00, 50, 1] } \\
{[\mathrm{THU}, 11: 00,50,1]}\end{array}$} & \multicolumn{2}{|c|}{ [(MON, TUE, THU) $(12: 30,13: 20)(30,60),(1,3)]$} \\
\hline & location & \multicolumn{2}{|c|}{$[\mathrm{CL}-100]$} & \multicolumn{2}{|c|}{ [RR-114] } \\
\hline & repetition- period & \multicolumn{2}{|c|}{$[09 / 18 / 2012,01 / 24 / 2013]$} & \multicolumn{2}{|c|}{$[09 / 18 / 2012,01 / 24 / 2013]$} \\
\hline \multirow{4}{*}{ Behavior Attributes } & attendance-rate & \multicolumn{2}{|c|}{0.95} & \multicolumn{2}{|c|}{0.7} \\
\hline & activity-arrival-trend & \multicolumn{2}{|c|}{$\begin{array}{l}\text { Normal }(-10 \%, 5 \%) \\
\text { Normal }(-5 \%, 2 \%)\end{array}$} & \multicolumn{2}{|c|}{ Uniform $(-10 \%, 10 \%)$} \\
\hline & activity-leaving -trend & \multicolumn{2}{|c|}{$\begin{array}{l}\text { Normal }(2 \%, 5 \%) \\
\text { Normal }(2 \%, 2 \%)\end{array}$} & \multicolumn{2}{|c|}{ Uniform $(-10 \%, 10 \%)$} \\
\hline & preferred-shorter-free-time & \multicolumn{2}{|c|}{ nil } & \multicolumn{2}{|l|}{ Y } \\
\hline
\end{tabular}

\section{B. Occupants' Movement Analysis}

Based on the activity timetable, the analysis of occupants' movement involves four steps, i.e., movement pattern determination, pattern decomposition, pattern counting, and conditional probability transforming, as shown in Fig. 1.

\section{Function-Space Assignment}

The optimization model maximizes the objective function under defined constraints by finding the best assignment of functions to spaces. The objective function used in this research is based on the concept proposed by Koopmans and Beckman [4]. Equation (1) is the objective function, which is a weighted average of two parts. First, $\left(X_{f i s i} \times P_{f i s i}\right)$ represents the assessment of the suitability of a function assigned to a space. For example, a classroom assigned to a large space is more suitable than to a small space. Secondly, $\left(X_{f i s i} \times X_{f j s j} \times\right.$ $\left.D_{s i s j} \times R_{f i j j}\right)$ represents the assessment of a function assigned to a space from the perspective of the moving distance $\left(X_{f i s i} \times\right.$ $\left.D_{\text {sisj }}\right)$ based on the movement relation $\left(X_{f j s j} \times R_{f i f j}\right)$. For example, strong related functions assigned to neighboring spaces may have a higher assessment value than that to spaces at a distance.

$$
\operatorname{Max} O=W_{1}\left(\frac{\sum_{f_{i}} \sum_{s_{i}} X_{f s_{i}} \times P_{f s_{i}}}{n}\right)+W_{2}\left(\frac{\sum_{f_{i}} \sum_{s_{i}} \sum_{f_{j}} \sum_{s_{j}} X_{f_{s i}} \times X_{f_{j, j}} \times D_{s, s_{j}} \times R_{f f_{j}}}{n}\right)
$$

Subjected to

$$
\begin{aligned}
& X_{f_{j} s_{i}}=0 \text { if } X_{f_{i} s_{i}}=1 \text { and } f_{j} \neq f_{i}, \\
& X_{f_{i} s_{j}}=0 \text { if } X_{f_{i} s_{i}}=1 \text { and } s_{j} \neq s_{i} .
\end{aligned}
$$

where $O=$ objective function; $X_{f i s i}=$ permutation matrix variable (the value is 1 if function $f_{i}$ is assigned to space $s_{i}$, and is 0 if not assigned to $\left.s_{i}\right) ; P_{f i s i}=$ suitability preference of function $f_{i}$ assigned to space $s_{i} ; D_{s i s j}=$ distance between spaces $s_{i}$ and $s_{j} ; R_{f i f j}=$ movement relation of functions $f_{i}$ and $f_{j}$ (the value is between 0 and 1 , where 0 represents no sequential movement pattern exists between functions $f_{i}$ and $f_{j}$, and 1 represents the use of $f_{i}$ always followed by the use of $f_{j}$ ); $n=$ total number of functions; $W_{l}, W_{2}=$ the weights between 0 and 1 .

\section{ACTIVITy Simulation AND FunCTION-SpaCE OPTIMIZATION}

\section{A. The Process Model for Activity Simulation}

The activity simulation deals with the fixed activities before the unfixed activities because most occupants put the fixed activity on the schedule first, then use the left free time for their unfixed activities. As shown in Fig. 2, the process is consisted of 12 primary steps, described as follows.

\section{B. Steps $1-2$}

The group activity list defines all the groups of occupants in the building and the associated activities. For each group, the model repetitively generates a member and its associated activities until the number of members reaches the designated number of members for the group. For each member, the process continues to determine the fixed activities and unfixed activities.

\section{Steps 3-4}

For each fixed activity, the model checks its repetition 
period, and only deals with the repetition period that falls within the desired simulation time period. Because the schedule-specs. (i.e., activity start time and duration) and location of a fixed activity are deterministic and cannot be changed, the process stops and produce a warning if a conflict is found. Step 3 is repeated until all the weekly schedules of the simulation time period are determined.

\section{Step 5}

Step 5 determines the actual values for the behavior attributes, which are probabilistic, to describe how a member participates in this activity, including the attendance-rate, activity-arrival-trend, and activity-leaving-trend. The process repeats Steps 3-5 until the simulated time schedule of all fixed activities associated with the member is completed.

\section{E. Step 6}

For each unfixed activity, the model checks its repetition period, and only deals with the repetition period that falls within the desired simulation time period.

\section{F. Steps 7-8}

For each unfixed activity occurrence, the model randomly determines how many times the unfixed activity should occur in a week.

\section{G. Steps 9-11}

If the preferred-shorter-free-time is YES, the process first randomly determine the activity duration and location, and then attempt to find the shortest free time currently available that can still accommodate the activity. Under the constraint set by the user-defined maximum number of trials, the attempt continues until a successful time slot is found.
If the preferred-shorter-free-time is $\mathrm{NO}$, the process first randomly determines the values of schedule-specs and location, then check if the schedule and location conflict with any other activities that have been already arranged. Similarly, under the constraint set by the user-defined maximum number of trials, the attempt continues until a successful unfixed activity is found.

\section{H. Step 12}

Step 12 generates the behavior attributes of the unfixed activity, which is the same as Step 5. The loop continues until all multiple occurrences of the unfixed activity have been determined. The process continues with the following week until the end of simulation time or repletion period, whichever is the smaller. This concludes the individual time table for the member for entire simulation time. The process continues for each member of the group, and for each group, until the timetables of all group members are determined. The final outcome of the simulation process is the activity timetable consisted of all individual time tables. To maintain the uncertain nature of activity participation, the activity simulation model creates an activity timetable for each occupant based on the probabilistic functions assigned to the occupant groups where he/she belongs. The function-space assignment model analyzes the timetables of all samples, and assigns the functions to the spaces under the specified constraints so that the total moving distance for the samples to participate in their activities is the minimum.

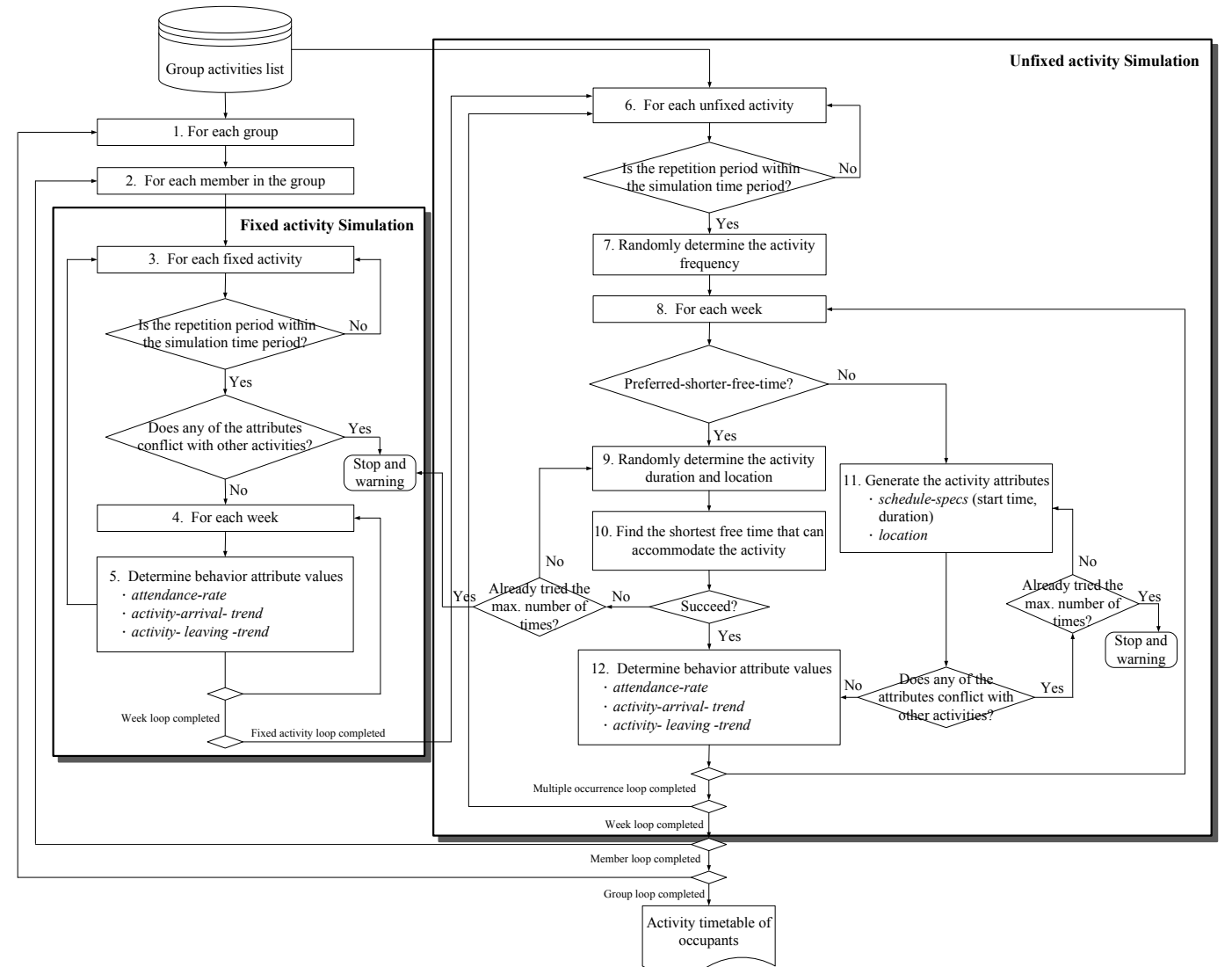

Fig. 2. The process model for activity simulation. 


\section{The Process Model for Function Assignment Optimization}

The function-space assignment is optimized using fmGA. The fmGA process is consisted of inner and outer loops. Each inner loop is called an era, and each outer loop is called an epoch. Thus, the execution of maximum number of eras defined by the era_max completes an epoch. The execution of the maximum number of epoch defined by epoch_max, terminats the fmGA evolution process.

The inner loop is consisted of three phases [9]: (1) the initialization phase - a population with sufficient chromosomes is created to contain all possible building blocks (BBs) of the order $\mathrm{k}$, where $\mathrm{BBs}$ refer to partial solutions of a problem; (2) primordial phase — bad genes are filtered out to maintain only the chromosomes with good fitness; and (3) juxtapositional phase-those good alleles (BBs) are rebuilt by cut-splice and mutation operations to form a high quality generation which tends to generate an optimal solution.

\section{CASE Study}

We conducted an experiment based on a real case to prove the research concept and to evaluate the performance of the proposed model. The following sections first introduce the case and then present the result of function-space assignment.

\section{A. Case}

The case used in this experiment is the building of the Civil Engineering Department of National Chiao-Tung University in Hsinchu, Taiwan. The building is a 4-story courtyard building with a total floor area of $6,616 \mathrm{~m}^{2}$. The experiment only used 10 key spaces (numbered from $s_{1}$ to $s_{10}$ ) as case study, consisting of an administration office $\left(f_{6}\right)$, a library $\left(f_{1}\right)$, a seminar room $\left(f_{3}\right), 2$ laboratories $\left(f_{8} \& f_{9}\right), 2$ meeting rooms $\left(f_{4} \& f_{5}\right)$, and 3 classrooms $\left(f_{2}, f_{7} \& f_{10}\right)$, situated separately at 4 different floors of the building. The 10 spaces can be divided into 3 groups, i.e., large (L: 90-135 $\mathrm{m}^{2}$ ), medium (M: 45-80 $\mathrm{m}^{2}$ ), and small $\left(\mathrm{S}: 15-20 \mathrm{~m}^{2}\right)$ spaces. Table $\mathrm{V}$ provides the types of space size and functions' preferred size. The simulation inputs were determined by the department renovation project manager with our facilitation.

TABLE V: SpaCE Size AND FunCTIONS' PREFERRED Size.

\begin{tabular}{|c|c|c|c|c|c|c|c|c|c|c|c|}
\hline \multicolumn{2}{|c|}{ Space } & \multicolumn{2}{|l|}{$s_{1}$} & $s_{2}$ & $s_{3}$ & $s_{4}$ & $s_{5}$ & $s_{6}$ & $s_{7}$ & $s_{8}$ & $s_{10}$ \\
\hline \multicolumn{2}{|c|}{ Size } & \multicolumn{2}{|l|}{$\mathrm{M}$} & $\mathrm{M}$ & $\mathrm{L}$ & $\mathrm{M}$ & $\mathrm{S}$ & $\mathrm{L}$ & $\mathrm{M}$ & $\mathrm{L}$ & $\mathrm{L}$ \\
\hline \multicolumn{2}{|c|}{ Function } & \multicolumn{2}{|l|}{$f_{1}$} & $\overline{f_{2}}$ & $\overline{f f_{3}}$ & $f_{4}$ & $f_{5}$ & $f_{6}$ & $f_{7}$ & $f_{8}$ & $f_{10}$ \\
\hline \multicolumn{2}{|c|}{ Preferred size } & \multicolumn{2}{|l|}{$\mathrm{L}$} & $\mathrm{L}$ & \multirow{2}{*}{$\begin{array}{c}\mathrm{L} \\
\text { Seminar } \\
\text { room } \\
\end{array}$} & $\mathrm{S}$ & $\mathrm{S}$ & $\mathrm{M}$ & $\mathrm{M}$ & M $\mathrm{M}$ & $\mathrm{L}$ \\
\hline \multicolumn{2}{|c|}{ Description } & \multicolumn{2}{|c|}{ Library } & Class- room & & \multicolumn{2}{|c|}{$\begin{array}{c}\text { Meeting } \\
\text { room }\end{array}$} & $\begin{array}{l}\text { Adm. } \\
\text { office }\end{array}$ & $\begin{array}{l}\text { Class- } \\
\text { room }\end{array}$ & Lab. & $\begin{array}{c}\text { Class- } \\
\text { room }\end{array}$ \\
\hline & & & & BLE VI: RES & \multicolumn{5}{|c|}{ T OF FUNCTION-SPACE ASSIGNMENT } & & \\
\hline \multirow{3}{*}{ Result } & \multicolumn{8}{|c|}{ Function-space assignment } & \multirow{2}{*}{\multicolumn{3}{|c|}{ Objective value }} \\
\hline & \multirow{2}{*}{$s_{1}$} & \multirow{2}{*}{$s_{2}$} & \multirow{2}{*}{$s_{3}$} & \multirow{2}{*}{$s_{4}$} & \multirow{2}{*}{$s_{6}$} & \multirow{2}{*}{$s_{8}$} & \multirow{2}{*}{$s_{9}$} & \multirow{2}{*}{$s_{10}$} & & & \\
\hline & & & & & & & & & Value & Impr & ement \\
\hline \multirow{2}{*}{$\mathrm{A}_{0}$} & \multirow{2}{*}{$f_{8}$} & & & & & & & & & $\left(\mathrm{~A}_{0^{-}}\right.$ & $\left.{ }_{0}\right) / \mathrm{A}_{0}$ \\
\hline & & $f_{9}$ & $f_{3}$ & $f_{5}$ & $f_{10}$ & $f_{1}$ & $f_{4}$ & $f_{6}$ & 1.1047 & & $\%$ \\
\hline $\mathrm{R}_{\mathrm{S}}$ & $f$ & & $f_{-}$ & $f_{-}$ & $f$ & & & & 12682 & $\left(\mathrm{R}_{1-}\right.$ & $\left.{ }_{0}\right) / \mathrm{A}_{0}$ \\
\hline $\mathrm{K}_{1}$ & 18 & 19 & 10 & $\int 7$ & $J_{1}$ & $J_{2}$ & $J_{5}$ & $\sqrt{3}$ & 1.2002 & & $0 \%$ \\
\hline
\end{tabular}

\section{B. Function Assignment Results}

The parameters epoch_max and era_max are defined to 5 and 4, respectively. Table VI shows different function-space assignments suggested by the architect (row $\mathrm{A}_{0}$ ), the proposed model (row $\mathrm{R}_{1}$ ) versions, and their corresponding performances in terms of objective values. The $\mathrm{R}_{1}$ version has a $14.80 \%$ higher objective value than the architect's version. The functions are also assigned to the space sizes most-preferred by the administrator (e.g., $f_{l}$ is assigned to the large spaces of $s_{6}, f_{5}$ is assigned to the small spaces of $s_{9}$ ). Additionally, the functions having larger are also placed at least at the same floor (e.g., $f_{8} \& f_{9}, f_{7} \& f_{9}$ are on floor 4 ).

The fmGA took 16 seconds with the maximum generation equal to 20 . It actually required only 10 generations converging the optimal assignment $\left(\mathrm{R}_{1}\right)$. The performance is acceptable considering the problem has a combination size of $3,628,800$ (=10!). In addition, with 10 generations of 448 populations, the number of solutions searched for by the proposed model was $4,480(=448 \times 10)$, which was only $0.123 \%(=4,480 / 3,628,800)$ of the search space.

\section{CONCLUSIONS}

This research proposed function-space assignment optimization model based on activity simulation. This research uses two modules to optimize the function space assignment. The first module uses simulation technique to randomly generate activity data to accommodate the uncertain nature of activities. The second module uses the fmGA to optimize the function-space assignment. This paper also presents a real case study to demonstrate the use of the proposed model and compares its assignments with those generated by a renovation architect. The result shows a $14.80 \%$ higher objective value than the architect's version. Our future research will focus on experiments with other types of buildings and objectives, and on extending this model.

\section{ACKNOWLEDGMENT}

This work was partially supported by the National Science Council grants (NSC 99-2221-E-009-133-MY3). Their support is greatly appreciated. 


\section{REFERENCES}

[1] A. Ekholm and S. Fridqvist, "A concept of space for building classification, product modelling, and design," Automation in Construction, vol. 9, no. 3, pp. 315-328, May 2000.

[2] R. Choudhary, A. Malkawi, and P. Y. Papalambros, "Analytic target cascading in simulation-based building design," Automation in Construction, vol. 14, no. 4, pp. 551-568, Aug. 2005.

[3] R. S. Liggett and W. J. Mitchell, "Optimal space planning in practice," Computer-Aided Design, vol. 13, no. 5, pp. 277-288, Sep. 1981.

[4] T. C. Koopmans and M. Beckman, "Assignment problems and the location of economic activities," Econometrica, vol. 25, no. 1, pp. 53-76, Jan. 1957.

[5] R. E. Burkard, “Quadratic assignment problems," European Journal of Operational Research, vol. 15, no. 3, pp. 283-289, March 1984.

[6] P. M. Hahn and J. Jrarup, "A hospital facility layout problem finally solved," Journal of Intelligent Manufacturing, vol. 15, no. 55/6, pp. 1-11, Oct. 2000.

[7] D. E. Goldberg and J. H. Holland, "Genetic Algorithms and Machine Learning," Machine Learning, vol. 3, no. 2-3, pp. 95-99, Oct. 1988.

[8] D. E. Goldberg, B. Korb, and K. Deb, "Messy genetic algorithms: motivation, analysis, and first results," Complex Systems, vol. 3, no. 5, pp. 493-530, 1989

[9] D. E. Goldberg, K. Deb, H. Kargupta, and G. Harik, "Rapid, accurate optimization of difficult problems using fast messy genetic algorithms," in Proc. Fifth International Conference on Genetic Algorithms, San Mateo, 1993, pp. 56-64.

[10] S. Doherty and K. Axhausen, "A conceptual model of the weekly household activity-travel scheduling process," presented at the Network on European Communications and Transport Activities Research Euro-conference, Israel, 1998.

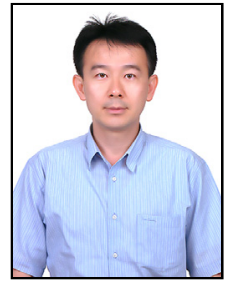

Ren-Jye Dzeng is a professor at the Department of Civil Engineering, National Chiao-Tung University, Taiwan. He is also acting as the CEO of the Eco-City: Smart Living Technologies Integrated Center, Taiwan. He received his Ph.D. from the Department of Civil \& Environmental Engineering, University of Michigan, Ann Arbor, USA. His research interests include construction engineering \& management, artificial intelligence, RFID, application of brain EEG and virtual reality for construction safety.

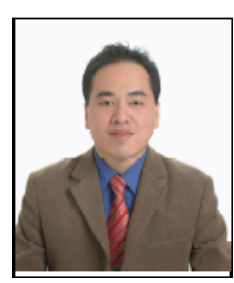

Chong-Wey Lin is an assistant professor at the Department of Communication and Technology, National Chiao-Tung University, Taiwan. He is also acting as the Coordinator of the Division of Open Innovation in the Eco-City: Smart Living Technologies Integrated Center, Taiwan. He received his Ph.D. Information Science in the Free University Berlin, Germany. His research interests include social networking and marketing, smart mobile service and cloud computing, innovation and entrepreneurship.

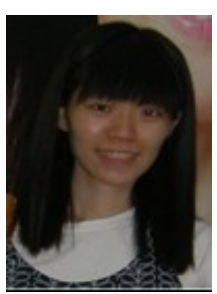

Fan-Yi Hsiao is a PhD Candidate at the Department of Civil Engineering, National Chiao-Tung University, Taiwan. She received her Master degree from the Department of Civil Engineering, National Chiao-Tung University, Taiwan. Her research interests include construction engineering \& management, artificial intelligence, and RFID application. 